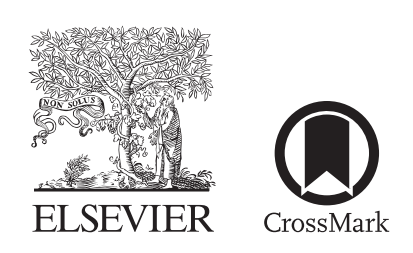

\title{
Results of One-Stage or Staged Amputations of Lower Limbs Consequent to Critical Limb Ischemia and Infection
}

\author{
Leandro Ramos Silva, Giordano Masini Fernandes, Natacha Ueda Morales, \\ Marcone Lima Sobreira, Regina Moura, Matheus Bertanha, and Winston Bonetti Yoshida, \\ Botucatu, São Paulo, Brazil
}

\begin{abstract}
Background: Amputations of lower limbs can be conducted as one-stage amputation (OSA) or staged amputation (SA) procedures. The objective of this study was to analyze technical success and mortality rates of both techniques, as well as factors that might influence outcomes in patients with critical limb ischemia (CLI).

Methods: A retrospective study of 185 consecutive patients with CLI who underwent amputations in the period 2004-2011. Primary end points were rates of technical success (healing without dehiscence or reintervention) and mortality. The influence on outcomes of demographic data, clinical status, and comorbidities was also analyzed by logistic regression.

Results: A total of 101 SA (91 patients) and 106 OSA (94 patients) were analyzed. SA had proportionally higher success rate (SA $77.2 \%$ vs. OSA $66.0 \%, P=0.0253$ ), lower perioperative mortality rate (SA, $10.9 \%$ vs. OSA, $20.7 \%, P=0.0247$ ), and lower 30 -day mortality rate (SA, $12.2 \%$ vs. OSA, $23.8 \%, P=0.0220$ ) in spite of more cases with Rutherford classes 5 and 6 (SA, $87.1 \%$ vs. OSA, $72.6 \%, P=0.0047)$, diabetes $(71.2 \%$ vs. $55.6 \%, P=0.0076)$, and infection (44.5\% vs. $28.3 \%, P=0.0061)$. Logistic regression demonstrated that in $S A$, success was more frequent in patients with diabetes who did not use insulin $(P=0.0072)$, in those with transfemoral amputations $(P=0.0392)$, with no coronary artery disease $(P=0.0053)$, and in foot infection $(P=0.0446)$, while for OSA success was more frequent in nondiabetic patients ( $P=0.0077)$, limbs without infection $(P=0.0298)$, amputations at foot level $(P=0.0155)$, or transfemoral amputations $(P=0.0030)$.

Conclusions: SA had a higher rate of technical success and lower mortality rates than OSA, even with greater number of patients with diabetes and more severe cases of ischemia and infection. However, prospective studies comparing both techniques are needed for further evidence.
\end{abstract}

\section{INTRODUCTION}

Atherosclerosis is the most frequent cause of heart disease, abnormalities of brain vessels, and peripheral arteries, as well as lower limbs amputations. ${ }^{1}$ Systemic arterial hypertension, dyslipidemia, diabetes mellitus, and smoking are important risk

Conflicts of interest: The authors have no conflicts of interest to disclose. Funding: Financial support provided by FAPESP (São Paulo State Research Supporting Foundation, Processes 2012/15563-8 and 2013/16724-8).

Department of Surgery and Orthopedics, Botucatu Medical School, Universidade Estadual Paulista, Campus of Botucatu, Botucatu, São Paulo, Brazil.

Correspondence to: Winston Bonetti Yoshida, PhD, Departamento de Cirurgia e Ortopedia da Faculdade de Medicina de Botucatu da factors for this condition and their control exerts strong influence over prognosis. ${ }^{1,2}$ Peripheral artery disease (PAD) is a severe chronic condition that affects mainly elderly patients with risk factors for atherosclerosis. ${ }^{1}$ Progress to critical limb ischemia (CLI), presenting with rest pain, gangrene, or ischemic ulcers, occurs in $5-10 \%$ of the cases and

Universidade Estadual Paulista, Campus de Botucatu, Botucatu, São Paulo 18618-970,Brazil;E-mail: winston@fmb.unesp.br

Ann Vasc Surg 2018; 46: 218-225

http://dx.doi.org/10.1016/j.avsg.2017.06.144

(c) 2017 Elsevier Inc. All rights reserved.

Manuscript received: April 7, 2017; manuscript accepted: June 24, 2017; published online: 6 July 2017 
can progress to amputation in 1.0 to $3.3 \% .^{2}$ In patients with diabetes, the risk for atherosclerotic arterial disease increases by 5-15 times and may be associated to severe limb infection; the presence of anaerobic organisms and gram-negative bacilli are predictors of limb loss. ${ }^{3,4}$ The occurrence of neuropathic trophic ulcer is a potential cause for devastating infections in deep compartments of the feet $^{3,5}$ and often goes unnoticed by the patient due to peripheral neuropathy.

Owing to the frequency and severity of PAD, a large number of patients come to Vascular Surgery Services in advanced stages of limb infection or present failure of previous arterial interventions, requiring amputation. ${ }^{4-7}$

An alternative for these patients is staged amputation (SA), where the first procedure is the guillotine in order to drain the infection, and the second a definitive, more proximal surgery, after infection control. ${ }^{8,9}$ This type of approach would have the advantages of reducing the risk of failure in the second amputation, lowering the rates of higher amputations, and the need for stump revision, with mortality rates similar to one-stage amputation (OSA). ${ }^{10,11}$ The other possibility is OSA. ${ }^{8}$

Few previous studies have compared such amputation strategies for patients with PAD. A nonrandomized study compared open, nonstaged, and staged amputations for minor amputations at the level of the forefoot, ${ }^{10}$ finding similar results with both techniques. In another study, nonstaged and staged techniques were randomized for below-knee amputations, and the authors found similar results for primary healing in 47 patients with CLI and foot infection, with no differences in the rates of dehiscence, infections, and reamputations. $^{11}$

The objective of this study was to analyze retrospectively the rates of success and mortality of SA and OSA, at any level of the lower limbs, as well as to evaluate the influence of risk factors, degree of ischemia, technical data in the success of the interventions, and mortality.

\section{METHODS}

This is a nonrandomized retrospective cohort study of consecutive cases with lower CLI submitted to SA or one-stage definitive amputation in our institution from 2004 to 2011 . Data covered the period of hospital stay and up to 12 months for mortality. The local internal review board approved the study. The diagnosis of CLI was based on the presence of rest pain, ischemic ulcers or gangrene in the extremities, and absence of peripheral pulses. Diabetic foot might have these symptoms plus abscesses, with or without distal pulses. Arteriography was used when there was doubt about the indication of primary amputation or about the level of amputation; computarized tomography angiography has limited availability in our institution. In cases of abscesses or devitalized tissue, drainage and/or debridement was carried out before the amputation, and all these patients received appropriate systemic antibiotics, considering that some rare patients could have a chance of limb preservation. The amputation level was decided based on clinical aspect (extensive necrosis, anaerobic ascending infection, and sepsis). Skin temperature and proximal pulses were adjunctive parameters. Measurements of skin perfusion were not performed.

\section{Inclusion Criteria}

(1) Patients diagnosed with PAD and CLI, with no palpable peripheral pulses in the foot and no possibility for or after failure of previous vascular surgery, whose final treatment was amputation of the compromised lower extremity; (2) Diabetic patients with severe gross infection, purulent drainage, and/ or neuropathic ulcers, with or without distal pulses.

\section{Exclusion Criteria}

Patients younger than 18 years; pregnancy; tumorrelated amputations; arteritis; occlusion consequent to acute arterial embolism or trauma; revisions of amputation stumps; different techniques in bilateral amputations; amputation of upper limbs.

\section{Indication of the Type of Amputation}

The SAs were primarily indicated in cases where necrosis was not well delimited, with abscesses or severe limb infections, osteomyelitis, and cellulitis. In this case, an open guillotine amputation was done in the foot or at transtibial distal levels, followed by closed amputation at a more proximal level after delimitation of the ischemia and necrotic area, and remission of the infectious process. OSAs were indicated for patients with well-defined area of critical ischemia, with no osteomyelitis/cellulitis or with infections that had been eradicated or were restricted to the foot. Indications out of this protocol could be carried out in special cases, due to medical or surgical risks, at the surgeon's discretion. 


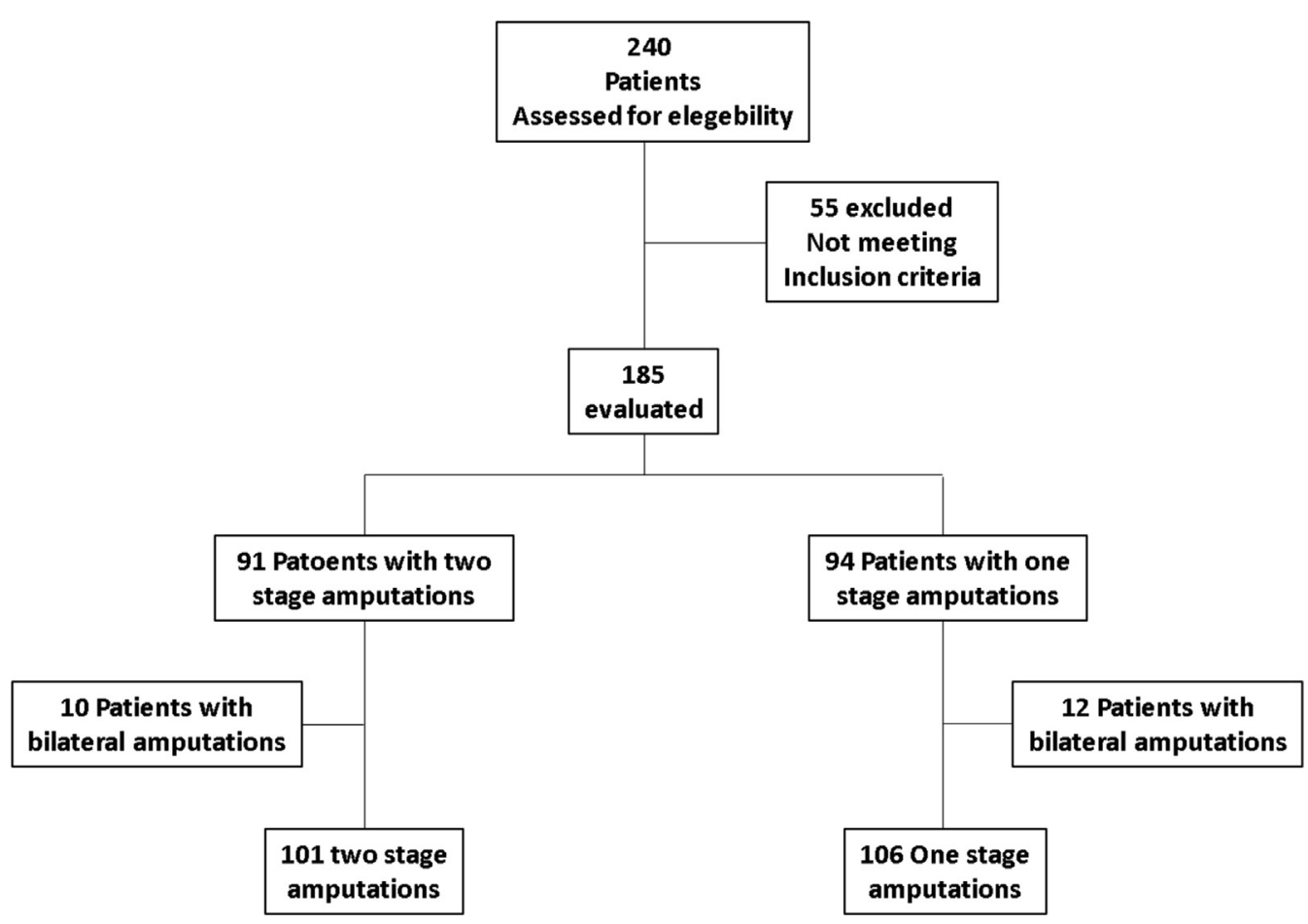

Fig. 1. Flowchart of included and excluded patients. Bilateral amputations were inclusion criteria, if they were performed with the same technique in both sides.

\section{Primary End Points}

Success was defined for non-SA as primary healing of the final amputation (no need for another surgery during the hospitalization period), that is, no stump revision or reamputation at any level within this period. For SAs, outcomes were similar to definitive closed amputations. For both types of amputation, failure was considered when patients had in the definitive amputation: suture dehiscence, stump ischemia, stump infection, and stump revisions in the same hospital stay. Infections were diagnosed by specialized vascular surgeons and confirmed by microbiology of the secretion culture. The other end points were in-hospital mortality (30 days) and late mortality (12 months) from any cause.

Variables that could potentially be associated with problems in amputation stumps healing such as smoking, cardiovascular and cerebrovascular history, severity of ischemia (Rutherford Classification), prior revascularization, level of amputation, infection and microbial flora, comorbidities (arterial hypertension, diabetes, neuropathy, coronary heart disease), level of the first amputation, and demographic data (gender, age, race, and affected side) were also collected. Smokers were defined as active smokers.

\section{Statistical Analysis}

Sample size was of convenience. The comparison of demographic data (proportional variables and outcomes between the 2 groups), was done using chisquared or Fisher's exact test. Continuous variables were analyzed by Student's $t$-test. Predisposing factors for outcomes were analyzed in each group separately, using a logistic regression model (categorical variables) or Cox model (quantitative variables), considering success and mortality in each group as the dependent variable, and the others as explanatory. In order to compare mortality between the 2 types of amputation, a Kaplan-Meier survival curve was used.

A 5\% significance level or the corresponding $P$ value was used in all tests. All analyzes were performed using SAS 9.3 Software system for Windows.

\section{RESULTS}

Medical records of 240 patients consecutively amputated between January 2004 and December 2011 were analyzed, of which 185 had PAD and CLI. Figure 1 depicts in detail the inclusion and 
Table I. Demographic data, risk factors, and main results of SA and OSA techniques

\begin{tabular}{|c|c|c|c|}
\hline Characteristics & $\begin{array}{l}\text { Staged amputation } \\
(\mathrm{SA} ; n=101)\end{array}$ & $\begin{array}{l}\text { One-stage amputation } \\
(\text { OSA; } n=106)\end{array}$ & $P$ value \\
\hline Success & $78(77.2 \%)$ & $70(66 \%)$ & $P=0.0253$ \\
\hline Mortality $<30$ days & $11(10.9 \%)$ & $22(20.7 \%)$ & $P=0.0247$ \\
\hline Mortality $1-12$ months & $11(12.2 \%)$ & $20(23.8 \%)$ & $P=0.0220$ \\
\hline Age (years) & $63.83 \pm 10.51$ & $64.08 \pm 10.75$ & $P=0.4329$ \\
\hline \multicolumn{4}{|l|}{ Gender } \\
\hline Male & $62(61.3 \%)$ & $61(57.5 \%)$ & \multirow[t]{2}{*}{$P=0.5739$} \\
\hline Female & $39(38.6 \%)$ & $45(42.4 \%)$ & \\
\hline Caucasian & $90(89.1 \%)$ & $96(90.5 \%)$ & \multirow[t]{2}{*}{$P=0.1711$} \\
\hline Others & $11(10.8 \%)$ & $10(9.4 \%)$ & \\
\hline Patients with diabetes & $72(71.2 \%)$ & $59(55.6 \%)$ & $P=0.0076$ \\
\hline Patients with diabetes using insulin & $26(25.7 \%)$ & $20(18.8 \%)$ & $P=0.1311$ \\
\hline Diabetic neuropathy & $21(20.7 \%)$ & $22(20.7 \%)$ & $P=0.1359$ \\
\hline Smokers & $64(63.3 \%)$ & $39(36.7 \%)$ & $P=0.0001$ \\
\hline Patients with hypertension & $71(70.2 \%)$ & $89(81.1 \%)$ & $P=0.0085$ \\
\hline Coronary disease & $22(21.7 \%)$ & $30(28.3 \%)$ & $P=0.0715$ \\
\hline Cerebrovascular disease & $14(13.8 \%)$ & $28(26.4 \%)$ & $P=0.0112$ \\
\hline Infection (G+ and/or $\mathrm{G}-$ ) & $45(44.5 \%)$ & $30(28.3 \%)$ & $P=0.0061$ \\
\hline \multicolumn{4}{|l|}{ Rutherford } \\
\hline$<3$ & $2(1.9 \%)$ & $4(3.7 \%)$ & \multirow[t]{6}{*}{$P=0.0047$} \\
\hline 4 & $11(10.8 \%)$ & $25(23.5 \%)$ & \\
\hline 5 & $30(29.7 \%)$ & $60(56.6 \%)$ & \\
\hline 6 & $58(57.4 \%)$ & $17(16 \%)$ & \\
\hline$<4$ & $13(12.8 \%)$ & $29(27.3 \%)$ & \\
\hline $5+6$ & $88(87.1 \%)$ & $77(72.6 \%)$ & \\
\hline \multicolumn{4}{|l|}{ Level of first amputation } \\
\hline Foot & $8(7.9 \%)$ & $6(5.6 \%)$ & $P=0.5174$ \\
\hline Malleolar & $7(6.9 \%)$ & $0(0 \%)$ & $P=0.0058$ \\
\hline Tibial & $75(74.2 \%)$ & $23(21.6 \%)$ & $P<0.0001$ \\
\hline Knee & $4(3.9 \%)$ & $2(1.8 \%)$ & $P=0.2237$ \\
\hline Thigh & $7(6.9 \%)$ & $75(70.7 \%)$ & $P<0.0001$ \\
\hline Previous revascularizations & $62(61.3 \%)$ & $75(70.7 \%)$ & $P=0.1543$ \\
\hline No revascularization & 39 & 31 & \\
\hline Length of stay (days) & $25.18 \pm 18.18$ & $16 \pm 16.3$ & $P=0.9999$ \\
\hline
\end{tabular}

exclusion algorithm of the subjects, showing a total of 91 cases with 101 SAs, and 94 cases with 106 OSAs. All patients included with bilateral amputations had the same amputation technique in both sides. The main outcomes and demographic data, as well as the distribution of clinical and surgical data are depicted in Table I. The technique used for definitive transtibial amputation was the posterior flap with suture in the anterior face of the stump (Fig. 2). A psychotherapist and a physiotherapist assisted all patients during hospital stay and rehabilitation after discharge. Standard dressing was used for both staged and closed amputations, washing the wound with saline, followed by gauze and bandage application.

Considering the demographic data (Table I), there were more patients with diabetes, smokers, and patients with infections in the SA group; moreover, these technique is indicated for more severe cases of ischemia and infection (Rutherford 6), consistent with our previously established protocol (Fig. 3). In the OSA group, there was a predominance of patients with hypertension and cerebrovascular disease. The length of stay was on average similar for both techniques (SA, $25.18 \pm 18.18$ days vs. OSA, 16.3 days $\pm 16 ; P=0.99991)$. The level of the first amputation was most often transtibial in SA and transfemoral in OSA (Table I).

Regarding primary end points (Fig. 4), SAs were more successful technically than one-stage procedures $(77.2 \%$ vs. $66.0 \%$, respectively; $[P=0.0253])$. The 30 -day mortality was significantly less frequent $(P=0.0247)$ in SA $(10.9 \%)$ than in OSA $(20.7 \%)$, as well as mortality rates in 


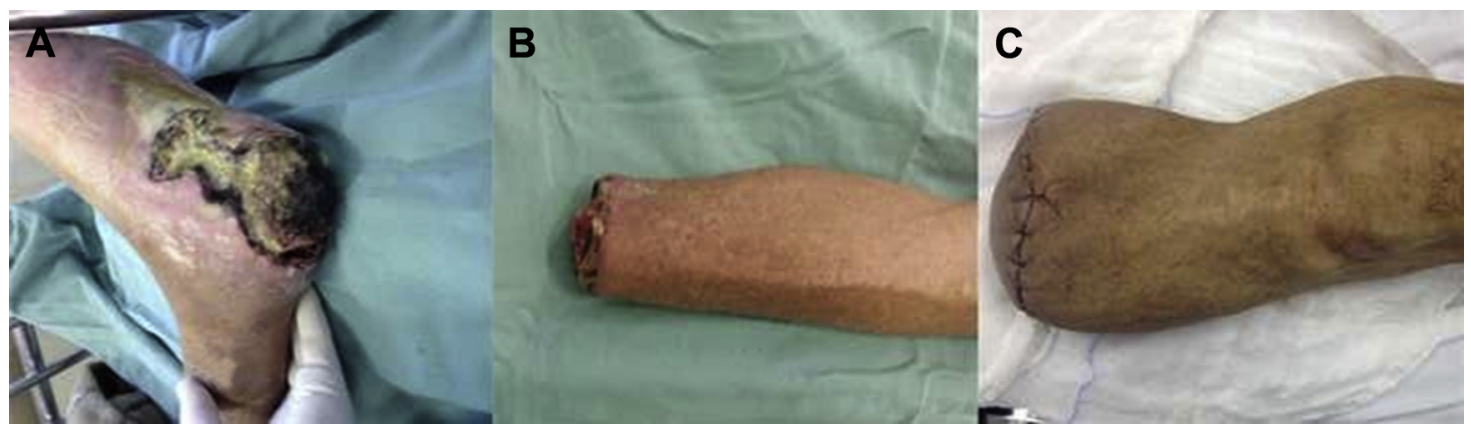

Fig. 2. Surgical aspects of SA preamputation foot (A), guillotine transtibial SA (B), and one-stage amputation (C).

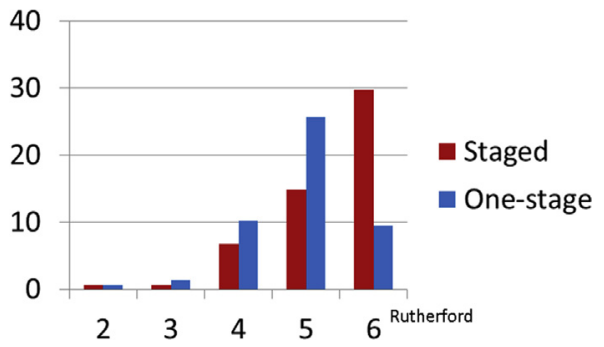

Fig. 3. Frequency of staged amputation (SA) and onestage amputation (OSA) according to Rutherford's classification.

the first year $(12.2 \%$ vs. $23.8 \%$, respectively; $[P=0.0220]$ ) (Table II).

In the separate analysis of the successful cases, success was more frequent in SA patients with Rutherford 5-6 $(P=0.0004)$ and transtibial amputations $(P=0.0001)$, while OSA success was more common in patients with hypertension $(P=0.0333)$, for patients without cerebrovascular disease $(P=0.0295)$, and in transfemoral amputations $(P=0.0001$; Fig. 5$)$. In successful SA, time interval median between the first and the final amputation was 7 days, and the mode was 3 days.

Considering success as dependent variable, logistic regression analysis of each type of amputation found that being classified as Rutherford 6 had no impact on the success rates in SA $(P=0.7209)$ but was significantly more frequent in diabetic patients without insulin $(P=0.0072)$ or using oral hypoglycemic agents $(P=0.0075)$, in infections restricted to the foot $(P=0.0044)$, and when the initial amputation was transfemoral $(P=0.0392)$. In OSAs alone, success also did not depend on the Rutherford classification and was more common in nondiabetic patients $(P=0.0077)$, in limbs with no gram-negative infection $(P=0.0298)$, amputations at foot level $(P=0.0155)$, or transfemoral $(P=0.0030)$. The other variables in both types of amputation did not

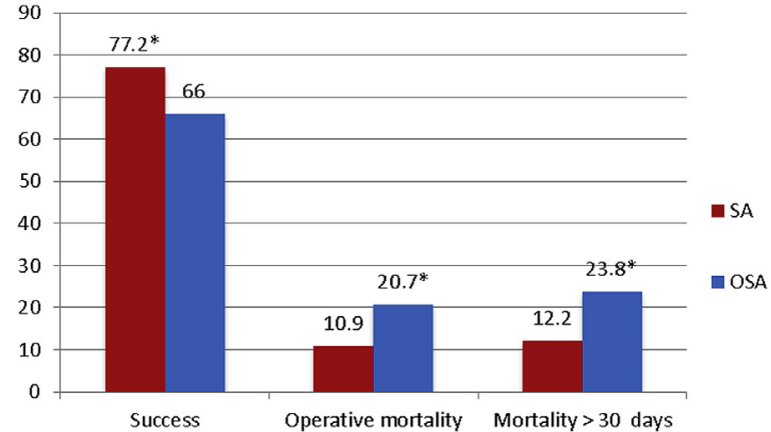

Fig. 4. Main outcomes after staged amputation (SA) and one-stage amputation (OSA). ${ }^{*} P<0.05$.

show statistical significance in the success or failure of the procedure.

Logistic regression was also used, considering operative survival as dependent variable ( $<30$ days). This analysis showed that the survival rate for OSAs was higher in patients with no coronary disease $(P=0.0492)$ and in transfemoral amputations $(P=0.0419)$. In SAs, there was no statistically significant variable influencing survival in this period.

When analyzing survival after 30 days (up to 1 year), logistic regression demonstrated that survival rate after OSA was higher in nondiabetic patients $(P=0.0101)$, in patients with diabetes with some treatment $(P=0.0476)$, and in nonsmokers $(P=0.0463)$. In SA, no variable impacted significantly on survival after 30 days.

Survival over time was statistically longer in patients with SAs (Fig. 6).

\section{DISCUSSION}

Success rate (Table I) was significantly higher in SA $(P=0.0253)$, even considering that SAs were mostly employed in patients with higher incidence of risk 
Table II. Success with SA and OSA, Rutherford's classification, comorbidities, and amputation levels

\begin{tabular}{|c|c|c|c|c|c|}
\hline & SA & OSA & Total & Hazard ratio/odds ratio & $P$ value \\
\hline Rutherford & & & & - & 0.0004 \\
\hline 2 & $1(0.68 \%)$ & $1(0.68 \%)$ & $2(1.35 \%)$ & & \\
\hline 3 & $1(0.68 \%)$ & $2(1.35 \%)$ & $3(2.03 \%)$ & & \\
\hline 4 & $10(6.76 \%)$ & $15(10.14 \%)$ & $25(16.89 \%)$ & & \\
\hline 5 & $22(14.86 \%)$ & $38(25.68 \%)$ & $60(40.54 \%)$ & & \\
\hline 6 & $44(29.73 \%)$ & $14(9.46 \%)$ & $58(39.19 \%)$ & & \\
\hline Hypertension & $54(37.76 \%)$ & $63(44.06 \%)$ & $117(81.82 \%)$ & $0.381(0.1535-0.9452)$ & 0.0333 \\
\hline Stroke & $11(7.53 \%)$ & $21(14.38 \%)$ & $32(21.92 \%)$ & $0.4092(0.1806-0.9272)$ & 0.0295 \\
\hline Ulcer & $31(20.81 \%)$ & $44(29.53 \%)$ & $75(50.34 \%)$ & $0.4047(0.2092-0.7829)$ & 0.0067 \\
\hline Foot level & $7(4.7 \%)$ & $1(0.67 \%)$ & $8(5.37 \%)$ & $6.9014(0.8274-57.5620)$ & 0.0377 \\
\hline Ankle level & $6(4.03 \%)$ & $0(0 \%)$ & $6(4.03 \%)$ & $12.8207(0.7090-231.8269)$ & 0.0187 \\
\hline Transtibial level & $58(38.93 \%)$ & $12(8.05 \%)$ & $70(46.98 \%)$ & $14.2583(6.3926-31.8026)$ & $<0.0001$ \\
\hline Transfemoral level & $3(2.01 \%)$ & $57(38.26 \%)$ & $60(40.27 \%)$ & $0.0098(0.0027-0.0358)$ & $<0.0001$ \\
\hline Dehiscence & $1(0.68 \%)$ & $0(0 \%)$ & $1(0.68 \%)$ & - & 0.0214 \\
\hline Infection & $7(4.76 \%)$ & $0(0 \%)$ & $7(4.76 \%)$ & & \\
\hline
\end{tabular}

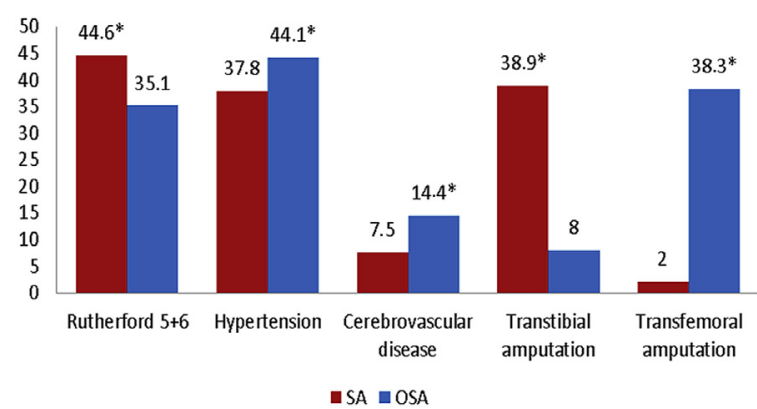

Fig. 5. Main factors influencing success in both amputation techniques. ${ }^{*} P<0.05$.

factors such as smoking and diabetes, and more severe ischemia and infection (Rutherford classification). Moreover, there were less early and late mortality rates in this group of patients, despite the worse clinical situation. As conclusion, both techniques were equally effective and safe, although success significantly predominated in SA, which could be considered a timely alternative in these severe cases. The level of amputation was significantly higher in OSA, corroborating 1 disadvantage of this technique pointed out by Berceli et al. ${ }^{10}$ In this study $(n=204)$, open amputations (for osteomyelitis, extensive necrosis, with no attempt for wound closure), SAs (minor toes osteomyelitis with proximal cellulitis, with open amputation of the foot, followed by wound closure 2-7 days later), and closed amputations (osteomyelitis confined to toes and foot bones, and minimal evidence of lymphangitis or tenosynovitis, with one-stage closed amputation) were compared. Their conclusion was that staged closure healed faster without negatively impacting the risk of major limb amputation. In the series by

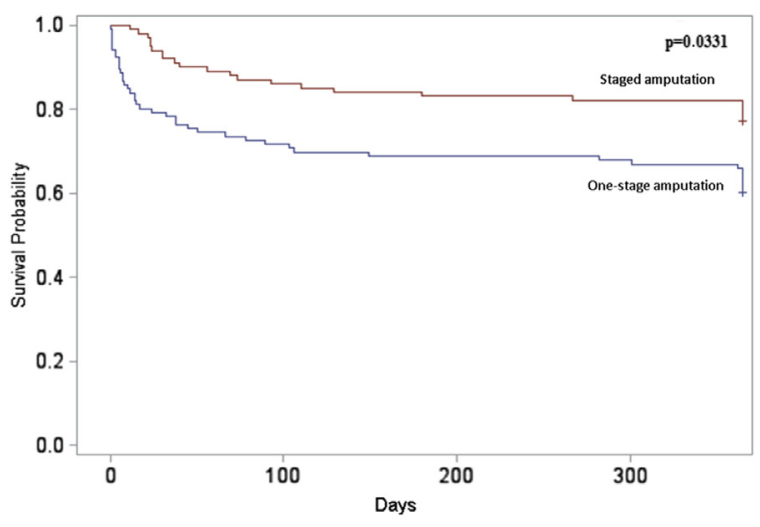

Fig. 6. Survival after staged amputation (SA) and onestage amputation (OSA).

Fisher et al., ${ }^{11}$ a comparison between one-stage and two-stage amputations showed that OSA had higher incidence of wound complications. In Altindas et al., 62 patients had SA after tibio-talar disarticulation, and they concluded that the risk of unnecessary tissue sacrifice and higher failure rate of the secondary transtibial amputation was reduced.

The surgical success of SA in our study was independent of the presence of diabetes mellitus but was more common in cases of less severe diabetes, in use of oral antidiabetic drugs and with no use of insulin. In OSA, success was favored by the absence of diabetes mellitus and infection. Patients with advanced diabetes often have impaired immunity, neurologic impairment, and microangiopathy, with more extensive and severe trophic lesions than nondiabetic patients. Success rates were greater with SAs in such cases, which is consistent with previous studies. ${ }^{10}$ 
Patients with hypertension had higher success rates only in OSA as compared with nonhypertensive patients $(P=0.0455)$. One would expect that hypertension would be responsible for more prominent atherosclerotic changes (since it is a significant risk factor), and consequently, for worse results, which was not the case in these patients. These results, however, have to be confirmed by other studies or larger sample series.

For both the techniques, the transfemoral level was more often successful than other levels of amputation. This is possibly due to the proximity of collateral circulation in this region via the deep femoral artery, as well as less trophic lesions and infections at this level. The ulcerations/trophic lesions at the foot level are more distant from the collateral circulation, which explains the negative correlation with success.

A systematic review demonstrated that the 30day mortality of limb amputations ranged between $4 \%$ and $22 \% .{ }^{12}$ A recent study showed $22 \%$ mortality in 30 days in amputated patients, $44 \%$ at 1 year, and $77 \%$ at 5 years $^{13}$; in our study, it was slightly higher at 30 days and lower at 1 year $(31.6 \%$ and $36.0 \%$, respectively).

Perioperative mortality ( $<30$ days) in this study was generally lower for SA, with no interference of the studied variables. In OSA, however, operative mortality was lower in transfemoral amputations and higher in patients with coronary disease, since amputation in these patients pose higher risk for both anesthesia and surgery. ${ }^{14}$ Transfemoral amputations, in addition to having greater collateral blood supply, are usually technically faster to accomplish. $^{15}$

The overall survival rate over time was greater in SA, corroborating isolated data of perioperative mortality and mortality after 30 days. In OSA, mortality after 30 days was higher in patients with diabetes mellitus in general, and especially in patients with diabetes taking some kind of antidiabetic medication, as well as in smokers. Smoking and diabetes contribute to cardiovascular complications and degenerative diseases, therefore reducing longevity. ${ }^{1,2,16}$

The major limitations of this study are the retrospective review, heterogeneity of cases and indications, noncomparative design, and also the election of the type of intervention being made at the surgeon's discretion. As a consequence, the indications for SAs tended to fall on patients with more advanced cases of infection and tissue impairment. The main objective of this study was to show success results separately for both techniques, as well as factors influencing success, using logistic regression. Demographic data in Table I was used just to show differences and similarities of both samples. On the other hand, it was a series from one single institution and team, during the same period, and with uniform management in relation to indications and surgical techniques. In addition, patients with all levels of amputation were included, and assessments were made of mortality and of the factors associated with success and mortality, characteristics that differentiate it from the only 2 previous studies that compared the 2 techniques. ${ }^{10,11}$

In conclusion, success was more frequent in SAs, even considering the higher incidence of risk factors such as smoking, diabetes mellitus, severity of ischemia, and infection in this group of patients. Furthermore, the operative and long-term mortalities were lower with this surgical technique (SA). Prospective randomized studies are needed to better define the advantages and limitations of OSA and SA techniques.

The authors acknowledge Associate Professor José Eduardo Corrente, PhD, Department of Biostatistics of the Botucatu Biosciences Institute/UNESP for the statistical analysis.

\section{REFERENCES}

1. Bhatt DL, Steg PG, Ohman EM, et al. International prevalence, recognition, and treatment of cardiovascular risk factors in outpatients with atherothrombosis. JAMA 2006;295: $180-9$.

2. Norgren L, Hiatt WR, Dormandy JA, et al. Inter-society consensus for the management of peripheral arterial disease (TASC II). J Vasc Surg 2007;45:5-67.

3. Zgonis T, Roukis TS. A systematic approach to diabetic foot infections. Adv Ther 2005;22:244-62.

4. Gamba MA, Gotlieb SL, Bergamaschi DP, et al. Lower extremity amputations in diabetic patients: a case-control study. Rev Saude Publica 2004;38:399-404.

5. Caiafa JS, Castro AA, Fidelis C, et al. Atenção integral ao portador de pé diabético. J Vasc Bras $2011 ; 10: 1-32$.

6. Heyer K, Debus ES, Mayerhoff L, et al. Prevalence and regional distribution of limb amputations from 2006 to 2012 in Germany: a population based study. Eur J Vasc Endovasc Surg 2015;50:761-6.

7. Kolossváry E, Ferenci T, Hováts L, et al. Trends in major lower limb amputation related to peripheral arterial disease in Hungary: a Nationwide Study (2004-2012). Eur J Vasc Endovasc Surg 2015;50:78-85.

8. Tisi PV, Than MM. Type of incision for below knee amputation. Cochrane Database Syst Rev 2014;4:1-30.

9. Altindas M, Kilic A, Cinar C. A reliable surgical approach for the two-staged amputation in unsalvageable limb and life threatening acute progressive diabetic foot infections: tibiotalar disarticulation with vertical crural incisions and secondary transtibial amputation. Foot Ankle Surg 2011;17: $13-8$. 
10. Berceli SA, Brown JE, Irwin PB, et al. Clinical outcomes after closed, staged, and open forefoot amputations. J Vasc Surg 2006;44:347-51.

11. Fisher DF Jr, Clagett GP, Fry RE, et al. One-stage versus twostage amputation for wet gangrene of the lower extremity: a randomized study. J Vasc Surg 1988;8:428-33.

12. Van Netten JJ, Fortigton LV, Hinchliffe RJ, et al. Early postoperative mortality after major lower limb amputation: a systematic review of population and regional based studies. Eur J Vasc Endovasc Surg 2016;51:248-58.

13. Fortington LV, Geertzen JHB, Van Netten JJ, et al. Short and long term mortality rates after a lower limb amputation. Eur J Vasc Endovasc Surg 2013;46:124-31.
14. Fleisher LA, Fleischmann KE, Auerbach AD, et al. 2014 ACC/AHA Guideline on perioperative cardiovascular evaluation and management of patients undergoing noncardiac surgery: A report of the American College of Cardiology/ American Heart Association Task Force on Practice Guidelines. J Am Coll Cardiol 2014;64:e77-137.

15. Eidt JF, Kalapatapu VR. Amputation techniques and results. In: Cronenwett JL, Johnston KW eds. Rutherford's Vascular Surgery. 7th ed. Philadelphia: Elsevier, 2010. pp 1772-91. Chapter 115.

16. Roglic G, Unwin N, Bennett PH, et al. The burden of mortality attributable to diabetes: realistic estimates for the year 2000. Diabetes Care 2005;28:2130-5. 